Exercise Intolerance and

Muscle Contracture 
Springer-Verlag France S.A.R.L 
G. Serratrice, J. Pouget, J.-Ph. Azulay (Eds)

\section{Exercise Intolerance and \\ Muscle Contracture}

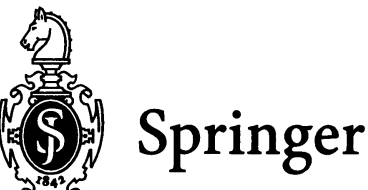


Georges Serratrice MD, FRCP

Professor of Neurology

Service de Neurologie et des Maladies Neuromusculaires

Centre Hospitalier Universitaire de la Timone

Marseille - France

Jean Pouget, MD

Professor of Neurology

Service de Neurologie et des Maladies Neuromusculaires

Centre Hospitalier Universitaire de la Timone

Marseille - France

Jean-Philippe Azulay, MD

Professor of Neurology

Service de Neurologie et des Maladies Neuromusculaires

Centre Hospitalier Universitaire de la Timone

Marseille - France

ISBN 978-2-287-59669-8

ISBN 978-2-8178-0855-0 (eBook)

DOI 10.1007/978-2-8178-0855-0

(C) Springer-Verlag France 1999

Originally published by Springer-Verlag France, Berlin, Heidelberg, New York in 1999

Library of Congress Cataloging-in-Publication Data

Exercise Intolerance and muscle contracture / [edited by] G. Serratrice, J. Pouget, J.-P. Azuley

P. cm.

Includes bibliographical references and index.

1. Contracture (Pathophysiology) 2. Exercise-Pathophysiology.

I. Serratrice, Georges. II. Pouget, J. (Jean), 1950-. III. Azulay, J.-P. (Jean-Philippe), 1960-.

[DNLM: I. Contracture-physiopathology. 2. Exercise Tolerance-physiology. 3. Muscle, Skeletal-physiopathology. WE 545 E96 1999]

RC925.6.E94 1999

616. 7'407--dc21

DNLM/DLC

for Library of Congress

99-27078

CIP

Apart from any fair dealing for the purposes of the research or private study, or criticism or review, as permitted under the Copyright, Designs and Patents Act 1988, this publication may only be reproduced, stored or transmitted, in any form or by any means, with the prior permission in writing of the publishers, or in the case of reprographic reproduction in accordance with the terms of licences issued by the copyright Licensing Agency. Enquiry concerning reproduction outside those terms should be sent to the publishers. The use of registered names, trademarks, etc. in this publication does not imply, even in the absence of a specific statement, that such names are exempt from the relevant laws and regulations and therefore free for general use.

Product liability: the publisher can give no guarantee for information about drug dosage and application there of contained in this book. In every individual case the respective user must check its accuracy by consulting other pharmaceutical literature. 


\section{Preface}

The main themes presented in this volume are exercise intolerance and muscle contracture. These two topics could seem different at first sight but share common clinical features. For instance, symptoms of exercise intolerance are myalgias and cramps but metabolic contractures as well. Likewise exercise intolerance is sometimes due to dystrophinopathy, itself causing contractures. Therefore, it is justified to gather these two syndromes in one study.

Exercise intolerance and muscle contracture could seem wellknown, even obsolete. It is not right. A reappraisal of these has become necessary for two reasons. First of all there are many new causes of exercise intolerance; secondly, the pathophysiology remains obscure in particular for muscle contracture and a clear classification is probably possible.

There is no book devoted to exercise intolerance and muscle contracture. This volume is divided in two parts related to the above topics. The two sections are introduced by a didactic and general overview, describing the main symptoms, classification and classical etiology. The other chapters, written by the best experts, present the recent advances in these syndromes and show many new aspects: new glycogenosis or mitochondriopathies, dystrophinopathies, malignant hyperthermia, myotonic disorders. Pathophysiology and classification of persistent contractures are described as well as therapy. Each chapter is thoroughly referenced, representing important progress in research in the field. Therefore, this comprehensive and updated volume allows neurologists, specialists in internal medicine, rheumatologists, pathologists, biochemists, physiotricians and all researchers interested in neuromuscular diseases to become familiar with many new and homogen data concerning exercise intolerance and muscle contracture which could really be considered as new syndromes. Moreover, this book will provide the basis for further developments. Most of the chapters have been presented in Marseille, France, on march 21st and 22nd 1998, during the XIV International Meeting on Neuromuscular Diseases. 


\section{Contributors}

ANDreu A.L.

H. Houston Merritt Clinical Research Center

Columbia University College of Physicians and Surgeons

630 West 168th Street

New York,

NY 10032, USA

Arahata K.

Department of Neuromuscular Research

National Institute of Neurosciences

National Center of Neurology and Psychiatry

Tokyo 187-8502, Japan

\section{Aubert M.}

Department of Anaesthesia and Intensive Care Unit

Hôpital d'Instruction des Armées

Laveran

13998 Marseille, France

\section{Azulay J.-Ph.}

Service de Neurologie et des Maladies Neuromusculaires CHU de la Timone

13005 Marseille, France

\section{Bartoli C.}

Laboratoire de Biopathologie Nerveuse et Musculaire, et Service d'Anatomie Pathologique et de Neuropathologie Hôpital de la Timone, 13005 Marseille, France

\section{Beckmann J.S.}

Genethon, Evry, France 


\section{BEHAN W.M.H.}

University Department of Pathology

Western Infirmary

Glasgow G11 6NT, UK

Bendahan D.

Centre de Résonance Magnétique Biologique et Médicale

URA C.N.R.S. ${ }^{\circ} 6612$

Faculté de Médecine, 27, Bd Jean Moulin

13005 Marseille, France

\section{Bianco N.}

Laboratoire de Biopathologie Nerveuse et Musculaire, et Service d'Anatomie Pathologique et de Neuropathologie Hôpital de la Timone 13005 Marseille, France

\section{Bollini G.}

Consultation des Maladies Neuromusculaires de l'Enfant Service d'Orthopédie Pédiatrique Hôpital d'Enfants, CHU Timone 13385 Marseille, France

\section{Bruno C.}

H. Houston Merritt Clinical Research Center

Columbia University College of Physicians and Surgeons 630 West 168th Street

New York, NY 10032, USA

\section{Carrelet P.}

Consultation des Maladies Neuromusculaires de l'Enfant Service de Neurologie Pédiatrique Hôpital d'Enfants, CHU Timone 13385 Marseille, France

\section{Chabrol B.}

Consultation des Maladies Neuromusculaires de l'Enfant Service de Neurologie Pédiatrique

Hôpital d'Enfants, CHU Timone

13385 Marseille, France

\section{Comi L.I.}

Via del Pini, 101

80131 Napoli, Italy 
Cozzone P.J.

Centre de Résonance Magnétique Biologique et Médicale URA CNRS $n^{\circ} 6612$

Faculté de Médecine, 27 Bd Jean Moulin

13005 Marseille, France

\section{Deslangles O.}

Laboratoire de Biopathologie Nerveuse et Musculaire, et Service d'Anatomie Pathologique et de Neuropathologie Hôpital de la Timone 13385 Marseille, France

\section{Desnuelle C.}

Service de Rééducation Fonctionnelle,

Hôpital de l'Archet

06200 Nice, France

\section{Di Mauro S.}

H. Houston Merritt Clinical Research Center,

Columbia University College of Physicians and Surgeons

630 West 168th Street

New York, NY 10032, USA

\section{Dubas F.}

Service de Neurologie A

Hôpital Larrey

CHU Angers

49033 Angers, France

\section{FARDEAU M.}

I N S E R M 153, Institut de Myologie

Groupe Hospitalier La Salpêtrière

47, Bd de l'Hôpital

75651 Paris Cédex 13

\section{Figarella-Branger D.}

Laboratoire de Biopathologie Nerveuse et Musculaire, et Service d'Anatomie Pathologique et de Neuropathologie Hôpital de la Timone 13005 Marseille, France 


\section{Fujimoto S.}

National Institute of Neurosciences

Tokyo 160, Japan

Gastaut J.-L.

Service de Neurologie

Hôpital Sainte-Marguerite

13009 Marseille, France

\section{GrigGs R.C.}

University of Rochester

School of Medicine and Dentistry

Rochester, NY 14642, USA

\section{Hadjigeorgiou G.M.}

H. Houston Merritt Clinical Research Center

Columbia University College of Physicians and Surgeons

630 West 168th Street

New York,

NY 10032, USA

Haller R.G.

Neuromuscular Center

Institute for Exercise and Environmental Medicine of Presbytarian

Dallas Hospital

V A Medical Center and The University of Texas

Southwestern Medical Center

7232 Greenville Avenue

Dallas, TW 75231, USA

IshiKawa T.

Department of Pediatrics

Nagoya City

University Medical School

Nagoya 466, Japan

\section{JANZER R.C.}

Service de Neurologie et Institut de Pathologie

CHU Vaudois,

1101 Lausanne, Switzerland 


\section{KaKULAS B.A.}

Department of Neuropathology,

Royal Perth Hospital and the Australian

Neuromuscular Research Institute

University of Western Australia

Perth, Western Australia

\section{KARPATI G.}

Montreal Neurological Institute and Hospital

3801 University Street

Montreal, Quebec

Canada H3A 2B4

\section{KozaK-Ribbens G.}

CRMBM-CNRS UMR 6612

Faculté de Médecine

27, Bd Jean Moulin

13005 Marseille, France

\section{Kubo S.}

Department of Neuromuscular Research

National Institute of Neurosciences

National Center of Neurology and Psychiatry

Tokyo 160, Japan

\section{KunTzer T.}

Service de Neurologie et Institut de Pathologie CHU Vaudois

1101 Lausanne, Switzerland

\section{LAYZER R.B.}

University of California

San Francisco CA 94143 0114, USA

\section{MacLennan D.H.}

Montreal Neurological Institute and Hospital 3801 University Street

Montreal, Quebec

Canada H3A 2B4

\section{Meola G.}

Department of Neurology, University of Milan San Donato Hospital

San Donato Milanese, Milan, Italy 


\section{MontFort M.F.}

Laboratoire de Biopathologie Nerveuse et Musculaire, et Service d'Anatomie Pathologique et de Neuropathologie

Hôpital de la Timone

13005 Marseille, France

\section{MoXLey III, R.T.}

University of Rochester

School of Medicine and Dentistry

Rochester, NY 14642, USA

\section{Munsat T.L.}

Department of Neurology

Tufts University

Boston, MA 02111, USA

Nigro G.

Via del Pini, 101

80131 Napoli, Italy

NonaKa I.

National Center Hospital for Mental

Nervous and Muscular Disorders

Tokyo 187-8551, Japan

\section{Paquis V.}

Laboratoire de Génétique

Hôpital de l'Archet

06200 Nice, France

\section{Pellissier J.F.}

Laboratoire de Biopathologie Nerveuse et Musculaire, et Service d'Anatomie Pathologique et de Neuropathologie

Hôpital de la Timone

13005 Marseille, France

\section{Pénisson-Besnier I.}

Service de Neurologie A

Hôpital Larrey

CHU Angers

49033 Angers, France 


\section{Pouget J.}

Service de Neurologie et des Maladies Neuromusculaires

CHU Timone

13005 Marseille, France

\section{RICHARD I.}

Genethon

Evry, France

\section{Rowland L.P.}

Columbia Presbyterian, Medical Center

The Neurological Institute

710 West 168th Street

New York, NY 10032-2603, USA

\section{SAITo K.}

National Institut of Neurosciences

Tokyo 187-8502, Japan

\section{SANSONE V.}

Department of Neurology, University of Milan

San Donato Hospital

San Donato Milanese, Milan, Italy

Serratrice G.

Service de Neurologie et des Maladies Neuromusculaires

CHU de la Timone

13005 Marseille, France

\section{ShansKe S.H.}

Houston Merritt Clinical Research Center,

Columbia University College of Physicians and Surgeons

630 West 168th Street

New York, NY 10032, USA

\section{Trojaborg W.}

The Neurological Institute

Columbia Presbyterian, Medical Center

710 West 168th Street

New York, NY 10032-2603, USA

\section{Tsuchiya Y.}

Department of Neuromuscular Research

National Institute of Neuroscience

National Center of Neurology and Psychiatry

Tokyo 187-8502, Japan 
UYAMA E.

Department of Neurology

Kumamoto University School of Medicine

Kumamoto 860, Japan 


\section{Contents}

\section{Exercise Intolerance}

Exercise Intolerance: Classification and Semiology

G. Serratrice, J. Pouget, J.-Ph. Azulay............................................. 1

P-31 Magnetic Resonance Spectroscopy of Metabolic

Abnormalities in Pathological Fatigue

D. Bendahan, P.J. Cozzone

New Aspects of Metabolic Myopathies

S. diMauro, C. Bruno, A.L. Andreu,

G.M. Hadjigeorgiou, S. Shanske

Brody's Syndrome: a Disorder of Calcium Uptake by the Sarcoplasmic Reticulum

G. Karpati, D.H. MacLennan

Stiffness on Exercise: a Non Progressive Disorder of Muscle Function (Brody-Karpati's Syndrome)

T. KUNTZER, R.C. JANZER

Exercise Intolerance in Calpain Deficiency and in $\alpha$-Sarcoglycanopathy

I. Pénisson-Besnier, I. Richard, F. Dubas,

J.S. Beckmann, M. Fardeau

Exercise Intolerance and Mitochondriopathies

C. Desnuelle, V. Paquis

Dystrophinopathies in Exercise Intolerance

B.A. KaKULAS.

Dystrophinopathies and CPT Deficiency in 75 Patients

with Exertional Heat Stroke and Rhabdomyolysis

D. Figarella-Branger, M. Aubert, G. Kozak-Ribbens, N. Bianco,

C. Bartoli, M.F. Montfort, O. Deslangles, J. Pouget,

P.J. Cozzone, G. Serratrice, J.F. Pellissier. 
Muscular Metabolism and Contraction

in Exertional Heat Stroke

G. Kozak-Ribbens, M. Aubert, D. Figarella-Branger,

D. Bendahan, P.J. Cozzone

Heroin, Methadone, HIV and Myalgia

J.-L. Gastaut

Cardiorespiratory Correlations in Exercise Intolerance

G. Nigro, L.I. Comı

Fibromyalgia: Symptom or Disease?

R.B. LAYZER....

Is Chronic Fatigue Syndrome a Muscle Disorder?

W.M.H. BeHAN

Exercise Intolerance and Muscle Pain in Myotonic Disorders

R.C. Griggs, V. Sansone, G. Meola, R.T. Moxley III.

Exertional Compartmental Syndromes

R.G. HALLER

\section{Muscle Contracture}

Reconciling Language Differences in Describing States of Muscle Shortening: Muscle Contracture and Related Conditions

G. Serratrice, L.P. Rowland

Muscle Contracture: Physiology and Clinical Classification

L.P. Rowland, W. Trojaborg, R.G. Haller.

Pathologic Aspects of Muscle Contracture

B.A. KaKulas

The Enigma of Muscle Contractures

M. FARDEAU

Contractures in Emery-Dreifuss Syndrome

K. Arahata, S. Fujimoto, E. Uyama, T. Ishikama,

S. Kubo, Y. Tsuchiya, I. Nonaka

Arthrogryposis and Spinal Muscular Atrophy

T.L. Munsat .

Therapy of Muscular Contractures

B. Chabrol, P. Carrelet, G. Bollini

Subject Index 